\title{
Pé diabético: ações de prevenção e cuidado por médicos de família e comunidade
}

\author{
Diabetic foot: prevention and care actions by family and community doctors \\ Pie diabético: acciones de prevención y atención de los médico de familia y de la \\ comunidad
}

Beatriz Angélica Pereira Braga ${ }^{1}$, Pollyana Cristina dos Santos Ferreira1*, Maria Teresa de Assis Campos ${ }^{1}$.

\section{RESUMO}

Objetivo: Investigar as ações desenvolvidas por médicos de Equipes de Saúde da Família em relação aos pés de pacientes com Diabetes Mellitus (DM). Métodos: Pesquisa descritiva e exploratória, com abordagem qualitativa, realizada entre 2018-2019, e em que participaram 12 médicos de família e comunidade de um município de Minas Gerais. Para as entrevistas utilizou-se roteiro semiestruturado, foram audiogravadas e seguiu-se a análise de conteúdo temática. Resultados: Emergiram três categorias temáticas: ações gerais destinadas a indivíduos com DM; ações específicas em relação aos pés dos diabéticos e desafios e dificuldades enfrentadas para o desenvolvimento de ações. Evidenciou-se que os médicos buscam orientar os indivíduos com DM desde cuidados gerais até específicos relacionados aos pés, destacando a avaliação durante o exame físico e ações de educação em saúde. Porém, ressaltam dificuldades associados à execução das ações. Conclusão: Evidenciou-se que os médicos de família e comunidade referem atuar com vistas a garantir a integralidade do cuidado. Entretanto, mencionam que a interferência de fatores estruturais, organizacionais e relacionados ao paciente com DM acaba, muitas vezes, por impactar no resultado final do trabalho.

Palavras-chave: Diabetes mellitus, Pé diabético, Médicos de família, Atenção à saúde.

\begin{abstract}
Objective: To investigate the actions developed by doctors of Family Health Teams in relation to the feet of patients with Diabetes mellitus (DM). Methods: This is a descriptive and exploratory research, with a qualitative approach, carried out between 2018-2019, in which participated 12 family and community doctors, from a municipality in Minas Gerais. For the interviews, a semi-structured script was used, audio tapes were recorded and thematic content analysis was followed. Results: Three thematic categories emerged: general actions aimed at individuals with DM; specific actions in relation to the feet of diabetics and challenges and difficulties faced for the development of actions. In them, it was evidenced that doctors seek to guide individuals with DM from general to specific care related to the feet, highlighting the evaluation during the physical examination and health education actions. However, they highlight difficulties associated with the implementation of actions. Conclusion: It became evident that family and community doctors refer to work in order to guarantee comprehensive care. However, they mention that the interference of structural, organizational and related factors to the patient with DM often ends up impacting the final result of the work.
\end{abstract}

Key words: Diabetes mellitus, Diabetic foot, Physicians family, Health care.

${ }^{1}$ Universidade Federal do Triângulo Mineiro (UFTM), Uberaba - MG. *E-mail: pollyana.ferreira@uftm.edu.br

SUBMETIDO EM: 3/2021

ACEITO EM: 3/2021

PUBLICADO EM: 4/2021 


\section{RESUMEN}

Objetivo: Investigar las acciones desarrolladas por médicos de Equipos de Salud de la Familia en relación a los pies de pacientes con diabetes mellitus (DM). Métodos: Investigación descriptiva y exploratoria, con enfoque cualitativo, realizada entre 2018-2019, participaron 12 médicos de familia y comunitarios de un municipio de Minas Gerais. Para las entrevistas se utilizó un guión semiestructurado, se grabaron cintas de audio y se siguió un análisis de contenido temático. Resultados: Surgieron tres categorías temáticas: acciones generales dirigidas a personas con DM; acciones específicas en relación con los pies de los diabéticos y desafíos y dificultades para el desarrollo de acciones. En ellos, se evidenció que los médicos buscan orientar a las personas con DM de la atención general a la específica relacionada con los pies, destacando la evaluación durante la exploración física y las acciones de educación para la salud. Sin embargo, destacan las dificultades asociadas a la ejecución de las acciones. Conclusión: Se evidenció que los médicos de familia y comunitarios remiten al trabajo con miras a garantizar una atención integral. Sin embargo, mencionan que la interferencia de factores estructurales, organizativos y relacionados al paciente con DM muchas veces acaba impactando el resultado final del trabajo.

Palabras clave: Diabetes mellitus, Pie diabético, Médicos de família, Cuidado de la salud.

\section{INTRODUÇÃO}

Quase meio bilhão de pessoas entre 20 e 79 anos, em todo o mundo, vive com diabetes mellitus (DM). No ranking internacional, o Brasil tem ocupado a quinta posição, correspondendo a 16,8 milhões de indivíduos com o diagnóstico da doença (IDF, 2019).

Para essa população a um risco de $25 \%$ de se desenvolver o pé diabético, sendo que a presença de ulcerações nos pés precede $85 \%$ das amputações em membros inferiores (BRASIL, 2016), podendo culminar em maior número de morbimortalidade e ampliar os custos relacionados ao setor de saúde (SOMAYAJI R, et al., 2017). Ademais, pesquisadores verificaram que a taxa de mortalidade entre pessoas com complicações relacionadas ao pé diabético foi mais alta quando comparada com aqueles sem lesões em pés e com a população geral (AL-RUBEAAN K, et al., 2017).

No Brasil, o Ministério da Saúde refere ser responsabilidade da Atenção Básica o acompanhamento integral da pessoa com DM, incluindo a prevenção, o diagnóstico e o manejo dessa população (BRASIL, 2016). Entretanto, ainda que existam guias e diretrizes terapêuticas e mesmo diante das possíveis complicações decorrentes de úlceras em pés de pacientes diabéticos, muitos médicos as desconhecem ou não as utilizam na prática. Assim, muitas vezes, as ações de prevenção e cuidado não são pautadas nas necessidades do paciente, ocasionando um atendimento voltado para as complicações, de forma tardia e com custos elevados, balizados por uma medicina prioritariamente curativa e pouco preventiva (MONTIELJARQUÍN AJ, et al., 2017).

Revisão sistemática apontou que ações de prevenção para as primeiras úlceras plantares em diabéticos são praticamente inexistentes, mas passam a acontecer no intuito de evitar úlceras recorrentes, principalmente voltadas à orientação para monitoramento domiciliar dos pés pelo paciente, na avaliação da temperatura da pele e no uso de calçados apropriados (VAN-NETTEN JJ, et al., 2016).

Em relação às pesquisas, nacionais e internacionais, as ações da equipe de saúde direcionadas aos pés de usuários com DM abordam, normalmente, a atuação da enfermagem. Nesse ínterim, estudos que avaliem a atuação dos profissionais médicos no contexto da Atenção Básica no que se refere aos cuidados com os pés de indivíduos com DM são incipientes (AALAA M, et al., 2017; FEITOSA MNL, et al., 2017; KUMARASINGHE SA, et al., 2018).

Considerando as complicações que podem advir como consequência do pé diabético, é importante a avaliação integral do indivíduo com DM no âmbito da Atenção Básica, incluindo o rastreamento de risco para lesões nos pés. Essas ações podem favorecer a realização de um trabalho preventivo, melhores prognósticos, podendo minimizar os riscos de ulcerações e amputações em membros inferiores (DUTRA LMA, et al., 2018). 
Nesse contexto, este estudo tem por objetivo investigar as ações desenvolvidas por médicos de Equipes de Saúde da Família (ESFs) em relação aos pés de pacientes com DM.

\section{MÉTODOS}

Pesquisa descritiva e exploratória, com abordagem qualitativa. Os dados foram coletados por meio de entrevistas semiestruturadas, com roteiro composto por perguntas norteadoras e um questionário de caracterização dos entrevistados.

Foram convidados a participar: médicos inseridos em ESFs do município de Uberaba-MG e que atuavam ou tivessem experiência profissional no contexto da atenção básica por pelo menos seis meses. Considerando que as ações realizadas pelos médicos, normalmente, estão vinculadas a critérios como área de abrangência, o vínculo do profissional com instituições de ensino; a possibilidade de compor os programas governamentais, entre outras condições, optou-se por realizar um sorteio aleatório para realizar o convite para participação do entrevistado na pesquisa, tendo como base a lista com os nomes das 55 ESFs existentes no município (FONTANELLA BJB, et al., 2011).

O encerramento da coleta de dados se deu pelo critério de saturação de dados (FONTANELLA BJB, 2008). Assim, participaram 12 médicos de ESFs de Uberaba-MG. Ocorreram 11 recusas; três equipes não possuíam médico; e um profissional não foi encontrado para agendamento da entrevista.

As entrevistas foram coletadas entre outubro de 2018 e junho de 2019. Foram gravadas em áudio, após a assinatura do Termo de Consentimento Livre e Esclarecido (TCLE) pelos participantes, e transcritas na íntegra em editor de texto (PEREIRA LF, et al., 2017).

Posteriormente, realizou-se a análise de conteúdo dos dados, o que contribuiu para a compreensão do discurso por meio da constituição de categorias temáticas (SILVA AH e FOSSÁ MI, 2015). Ocorreram em três etapas: pré-análise, exploração do material e tratamento dos resultados (CAMPOS CJG, 2004).

Foram respeitados os preceitos éticos, como apresentação e assinatura do TCLE, e a preservação do anonimato. O projeto foi aprovado, pelo Comitê de Ética em Pesquisa com Seres Humanos da UFTM, com o parecer 2.827.776.

\section{RESULTADOS}

Predominou o sexo feminino (58,3\%), com idade entre 30 e 39 anos (58,3\%) e a grande maioria possuía especialização $(91,7 \%)$. Metade dos participantes relatou ter realizado residência médica, sendo que $83,3 \%$ na área de Medicina de Família e Comunidade; 50\% referiram ter especialização Lato sensu, e apenas um $(8,3 \%)$ possuía mestrado. A maioria $(66,6 \%)$ possuía de um a nove anos de experiência na Atenção Básica, com tempo de atuação na ESF atual variando de um a três anos $(41,7 \%)$.

A partir da análise dos dados, as unidades de significados foram organizadas sistematicamente emergindo três categorias temáticas: ações gerais destinadas a indivíduos com DM; ações específicas em relação aos pés dos diabéticos e desafios e dificuldades enfrentadas para o desenvolvimento de ações.

\section{Categoria temática I: ações gerais destinadas a indivíduos com DM}

Todos os participantes referiram desenvolver ações genéricas aos pacientes com DM, incluindo aspectos como a prescrição e acompanhamento do uso de medicamentos, a orientação sobre hábitos de vida, além da prevenção e avaliação de complicações oriundas do DM, como ilustra as falas a seguir:

"Bom, a gente primeiro tem o tratamento medicamentoso, e o tratamento não medicamentoso. $O$ tratamento não medicamentoso a gente sempre está orientando dieta saudável, exercício físico, as orientações gerais para diabético." (A3)

"Os cuidados são a preocupação em relação ao uso correto das medicações, o controle com os exames laboratoriais para os pacientes que estão bem controlados 
a cada 6 a 12 meses, a avaliação de complicações, que é a nefropatia, a retinopatia, a neuropatia diabética." (A9)

O grupo Hiperdia foi mencionado como ferramenta imprescindível no processo de atenção integral, em que é possível desenvolver ações diversificadas ao paciente com DM, sobretudo com vistas ao cuidado geral, como pode ser exemplificado nos excertos a seguir:

"[...] atualmente, junto com o grupo Hiperdia, por exemplo, nós desenvolvemos muitos trabalhos de educação em saúde, orientação e cuidado, e trabalhos ligados à adoção de medidas para tratamentos adequados." (A5)

[...] "mas a gente tem o dia do Hiperdia, que a gente atende os diabéticos e hipertensos. É um programa voltado para eles. Aí nesse dia eu corrijo as medicações que estão tomando, peço exame para avaliar a evolução da doença, a eficácia da medicação e aí de repente se tiver dando muito alterado a gente troca a medicação." (A8)

Nesse contexto, conforme os relatos supracitados, o papel do médico de família e comunidade vai para além do modelo prescritivo, podendo ser utilizadas ferramentas que contribuam para um atendimento holístico, com foco nas especificidades e de forma a abranger a prevenção, o diagnóstico e o acompanhamento.

\section{Categoria temática II: ações específicas em relação aos pés dos diabéticos}

As ações específicas destinadas aos pés de pacientes com DM também foram relatadas pelos entrevistados. De modo geral, em seu cuidado diário com os pacientes, todos mencionaram a orientação sobre os cuidados básicos, como higiene e autoavaliação dos pés, uso correto de calçados; o manejo do pé diabético, englobando a avaliação, tratamento de lesões e encaminhamento para outros níveis de atenção, quando necessário, conforme os trechos a seguir:

"A gente faz avaliação de pé, de calçados, de contexto de vida também" [...]. (A7)

[...] "orientação em relação à higienização, calçados, né, evitar umidade entre os dedos, e sobre qualquer lesão procurar a unidade básica." (A11)

[...] "os curativos quando tem indicação de antibióticoterapia, a gente faz aqui mesmo. [...] uma úlcera mais grave crônica de difícil cicatrização, a gente encaminha para o hospital [...], para o ambulatório de pé diabético, de ferida infectada." (A9)

"Sempre orientamos, questão do calçado, a questão do cuidado quanto à confiabilidade que eles têm na sua visão, que vai se deteriorando ao longo do tempo no paciente diabético. Orientamos muito quanto ao tipo de trabalho que o paciente executa e alguns hábitos que podem ser, ainda que passageiros, [como, por exemplo] a um trajeto pequeno do trabalho que vão fazer sem nenhum tipo de calçado, que isso conhecemos aumenta bastante o risco de lesões." (A5)

A participação da equipe multiprofissional da atenção básica e a parceria com a Universidade destacaramse entre os entrevistados, evidenciando da interação de múltiplos agentes na construção de um sistema de atendimento integral à saúde, sobretudo no acolhimento a pacientes com múltiplas complicações e necessidades singulares.

[...]" eu sempre peço ajuda aos enfermeiros. Eu acho que o enfermeiro, de um modo geral, ele faz bastante capacitações sobre feridas. Muitas vezes, coincide que o aluno que tá aqui fazendo estágio para enfermagem." (A10)

"Nós temos o trabalho do pessoal do NASF, a fisioterapeuta, por exemplo, do NASF executa um trabalho muito bacana, nós temos uma profissional da fisioterapia, (...), 
que lá exerce alguns trabalhos ajuda nessa detecção. Nós temos uma profissional da Nutrição, também vinculada à Universidade, que participa ativamente desse processo de busca. Nós temos lá alguns acadêmicos, conduzidos por profissionais da área de enfermagem e medicina, e que acabam fazendo trabalho de visita domiciliar." (A5)

Percebe-se que os entrevistados buscam realizar ações específicas que favoreçam os cuidados com os pés. Além disso, a participação da equipe multiprofissional foi destacada, evidenciando a importância da interação dos múltiplos agentes de saúde na integralidade do cuidado.

\section{Categoria temática III: desafios e dificuldades enfrentadas para o desenvolvimento de ações.}

Os profissionais relataram enfrentar uma série de desafios e dificuldades para o desenvolvimento das ações. Entre elas foi citada a ausência de capacitações promovidas pelo sistema de saúde a toda equipe, e não só ao médico, comprometendo o atendimento especializado aos pacientes.

"Eu acho que a capacitação dos profissionais seria essencial, porque, por exemplo, a gente vai ver então que a maioria dos profissionais que atuam na saúde da família aqui [...], eles não são especialistas, então tem que ter capacitação tá. E não sei, não seria só do médico. Do médico e do enfermeiro né. Eu, quando fiz faculdade não existia em momento nenhum, [...] eu não tinha nada voltado pé diabético." (A10)

"A gente precisa desde propaganda, desde palestra, desde capacitação de funcionários, e não é capacitação só do médico não, é a capacitação do médico, da enfermeira, da técnica e principalmente dos agentes comunitários." (A1)

A questão da infraestrutura é abordada pelos profissionais como um dos grandes entraves para a integralidade no cuidado com os pés dos pacientes diabéticos.

"A gente não tem material. Às vezes não tem nem gaze para fazer um curativo, às vezes não tem pomada para aplicar nesse curativo, a gente fica meio assim sem ter o que fazer. (A4)

"O difícil é a infraestrutura, ao material para fazer o exame que aqui gente não tem." (A9)

"Nós temos, frequentemente, dificuldade quanto à presença de insumos para fazer o gerenciamento de lesões. Apenas para exemplificar, é uso de soro fisiológico no manejo de lesões, (...) já algum tempo nós não temos tido abastecimento desse item, que é item básico." (A5)

Todos os entrevistados afirmaram não realizar a classificação de risco de modo sistematizado, apontando como justificativa a falta de capacidade técnica, limitações de infraestrutura das unidades, bem como a falta de tempo.

"Eu acho que a gente não teve esse costume. Além de não ter o costume, não ter o espaço adequado, a capacitação né. Porque você vê, tem alguns pacientes que se você for examinar ele na frente dos outros essa sala que não é uma sala apropriada né." (A10)

"Às vezes, quando dá tempo de fazer, porque a demanda aqui é muito grande, às vezes a gente faz, não é sempre." (A3)

"Nós não temos ainda sistematizado em nossa unidade a estratificação que hoje consta em literatura. Muitas vezes, essa estratificação acaba sendo empírica e ela inclusive está sendo cogitada como educação continuada para as próximas semanas." (A5)

Além disso, participantes, em sua totalidade, alegaram dificuldades quanto à adesão ao tratamento em relação ao paciente diabético. Especialmente em questões que envolvem a adoção de práticas de 
autocuidado, a adesão ao tratamento medicamentoso e aos bons hábitos de vida, mesmo diante da orientação constante.

"Tem um pessoal que ainda é resistente ao tratamento. Parece que usa assim um mecanismo de negação, que não tem a doença, não quer aceitar, não quer seguir as orientações." (A3)

"Até dificuldade do próprio paciente de aceitação da visita das meninas, da orientação, da aderência ao tratamento." (A6)

"Outra grande dificuldade de que dispomos é quanto à simplicidade do paciente, dificuldade de compreender o cuidado, nem sempre eles dão conta da gravidade do quadro." (A5)

Diante a esses fatos, a interação médico-paciente e o cuidado centrado na pessoa foram relatados como possibilidades de aproximação.

"Eu acho que tem uma questão que talvez a gente tenha que aprimorar: uma abordagem centrada na pessoa. Então assim, o que especificamente para aquele indivíduo vai fazer diferença para que ele tenha uma postura mais proativa, mais harmônica com a proposta terapêutica né". (A7)

\section{DISCUSSÃO}

O tratamento do DM possui a finalidade de diminuir as taxas de morbimortalidade. Nesse sentido, o seu manejo deve incluir, entre outros aspectos, controle glicêmico; modificações nos hábitos de vida; tratamento medicamentoso; controle de fatores de risco cardiovascular; detecção e tratamento precoce de complicações e avaliação de comorbidades associadas (REYES-GARCíA R, et al., 2019). Assim, a atenção básica tem papel fundamental, especialmente nas ações do médico de saúde da família e comunidade, na abordagem do indivíduo com DM, assim como mencionado pelos participantes do estudo.

A atenção integral ao paciente deve considerar os momentos de educação em saúde que ocorrem nos grupos, por possibilitarem a apropriação crítica do conhecimento pelos envolvidos, incluindo questões individuais que podem impactar no bem viver e nas próprias demandas do serviço de saúde (CRUZ PJSC, et al., 2018). O grupo Hiperdia parece ser o momento em que os médicos utilizam junto aos demais membros da equipe, para desenvolverem as atividades de educação em saúde.

Em relação ao grupo Hiperdia, Salci MA, et al. (2017) verificaram que o atendimento a pessoa com DM, geralmente, acontecia coletivamente, com o intuito de dinamizar o serviço dos profissionais e favorecer 0 acesso a uma quantidade maior de usuários com o mesmo diagnóstico, em dias e horários pré-estabelecidos. Observaram, ainda, que o modelo de assistência tinha como prioridade a consulta médica, na qual entre as principais atividades havia a entrega de receitas e, eventualmente, a solicitação de exames. Consoante ao identificado nesta pesquisa.

Por outro lado, grupos, como o HIPERDIA, podem contribuir para a atenção à saúde estimulando o protagonismo do indivíduo, voltados ao enfrentamento da doença e a prática de autocuidado (CRUZ PJSC, et al., 2018). Nessa perspectiva, o papel do médico de família e comunidade vai para além do modelo prescritivo, contribuindo para um atendimento holístico, com foco nas especificidades e de forma a abranger a prevenção, o diagnóstico e o acompanhamento.

Sobre os cuidados específicos direcionados aos pés dos pacientes com DM, a avaliação anual e a orientação sobre as formas de manejo com os pés, a educação em saúde, o tratamento imediato em caso de pacientes com lesões, além do encaminhamento a outros níveis de atenção são atribuições relacionadas ao médico da equipe, conforme relatado pelos entrevistados (BRASIL, 2016).

Ressalta-se que o uso de calçado inapropriado é uma das principais causas de ulceração, principalmente se o pé apresentar algum tipo de deformidade. Estudo realizado por Silveira DM (2017) e colaboradores 
obteve que $33 \%$ dos entrevistados usavam calçado inapropriado durante a entrevista, indicando a importância das orientações em relação ao cuidado com os pés. Esses dados corroboram a preocupação dos médicos entrevistados, os quais mencionaram realizar avaliação e orientar sobre os cuidados com os pés e sobre os calçados.

A classificação de risco para ulcerações nos pés de indivíduos com DM está incluída nas diretrizes para o manejo do pé diabético. Nesse sentido, é fundamental que os profissionais de saúde, na atenção primária, estejam capacitados para incorporar essa prática na rotina do atendimento (LUCOVEIS MLS, et al., 2018).

A partir dessa classificação é possível realizar um plano de intervenção individual, de maneira a prevenir o surgimento lesões nos pés. Porém, exige a realização sistemática de um exame físico minucioso tanto do ponto de vista vascular, quanto neurológico, especialmente nos membros inferiores (GARBEY DLC, et al., 2018).

Entretanto, mesmo reconhecendo como de atuação médica a classificação de risco, os entrevistados referiram não realizá-la parcial e/ou integralmente em suas atividades cotidianas, enfatizando a necessidade de capacitação técnica sobre o assunto. Ressalta-se, ainda, a falta de pesquisas sobre a atuação dos médicos da atenção primária a respeito da classificação de risco e quanto às medidas preventivas para o pé diabético, mesmo diante da relevância do tema.

A participação da equipe multiprofissional foi evidenciada com destaque, no que tange a interação dos múltiplos agentes de saúde na integralidade do cuidado. Consoante ao observado em pesquisa nacional, em que mesmo frente aos desafios vivenciados pelas equipes do NASF e da Atenção Básica a existência de uma equipe multiprofissional era considerada como aspecto positivo e que favorecia o planejamento integrado das ações, de modo individual e coletivo, permitindo a integralidade do cuidado (SÁ SCM, et al., 2021).

Estudo realizado em unidades de saúde, de um município de Minas Gerais, verificou que a capacitação disponibilizada aos profissionais acontecia de forma isolada e a depender de cada local. Os entrevistados relataram que a falta de capacitação pode impactar no processo de trabalho, no cuidado à pessoa com DM, repercutindo em encaminhamentos não produtivos (GAMA CAP, et al., 2017).

Desse modo, ressalta-se a importância de investimentos em capacitação profissional voltados ao paciente com DM, a partir de uma postura ativa, realizando práticas que estejam de acordo com os princípios do Sistema Único de Saúde (SUS), com vistas a enfrentar as adversidades que corroboram o desenvolvimento do pé diabético (GAMA CAP, et al., 2017).

A falta de recursos institucionais pode acabar acarretando no desenvolvimento de ações aquém do esperando e acabar influenciando negativamente no resultado do trabalho dos profissionais (BISPO JÚNIOR JP e MOREIRA DC, 2018)

Pesquisa realizada por Fernández IC e Rumbo-Prieto JM (2018) observou que pacientes atendidos na atenção primária possuíam baixo conhecimento sobre o autocuidado com os pés. Esse fato poderia estar relacionado, entre outras causas, pelo baixo nível de informações disponibilizadas pelos profissionais de saúde e/ou pelas condições socioculturais dos pacientes. No mesmo estudo, mais da metade dos pacientes afirmaram não ter sido oferecida educação em saúde a esse respeito. Em contrapartida, em nossa investigação, os profissionais relataram abordar durante as consultas questões envolvendo o autocuidado e a prevenção de complicações em relação aos pés, porém o nível sociocultural dos pacientes foi considerado um dos grandes desafios.

Pesquisas nacionais têm demonstrado adesão insatisfatória por pacientes com DM ao tratamento farmacológico, sendo ainda mais evidente ao não farmacológico, principalmente ao que se refere aos hábitos de vida, como alimentação e prática de atividade física (GOMES AC, et al., 2020; SANTOS AL, et al., 2020).

Investigação sobre adesão ao tratamento para DM demonstrou que uma postura hierarquizada na relação profissional-paciente, pautada apenas na transmissão de informações, imposição de condutas e nas definições do que se considera certo ou errado acaba por aumentar o distanciamento entre as partes, interferindo no resultado final e aumentando a resistência do paciente (SANTOS AL, et al., 2020). 
Em contrapartida, a escuta qualificada, a comunicação efetiva, a compreensão de fatores subjetivos do contexto em que estão imersas as pessoas com DM, incluindo a identificação da rede de apoio social e familiar, são habilidades essenciais a serem exploradas pelos profissionais de saúde inseridos na atenção primária (AMORIM MMA, 2019).

Portanto, torna-se indispensável que o médico de família e comunidade conheça a realidade do paciente, considerando suas condições socioeconômicas, percepções e expectativas em relação ao tratamento. Dar voz ao paciente, possibilitando relatar suas experiências e percepções sobre a própria condição, além do incentivo ao próprio cuidado, principalmente em relação aos pés, pode facilitar adesão ao tratamento, bem como fortalecer a relação médico-paciente na atenção básica.

\section{CONCLUSÃO}

Os resultados evidenciaram que os médicos das ESF no atendimento aos indivíduos com DM procuram orientá-los, desde cuidados gerais, como a avaliação e o tratamento do DM, até cuidados específicos relacionados ao pé diabético, como a avaliação durante o exame físico, o manejo das lesões e ações de educação em saúde. Porém, possuem algumas dificuldades e desafios envolvendo a execução das ações, como a falta de insumos, infraestrutura das unidades, falta de capacitações a serem fornecidas pela rede de saúde, assim como dificuldades na adesão ao tratamento por parte dos pacientes. Ainda assim, o presente estudo pode contribuir para ampliar o conhecimento sobre a temática, uma vez que a abordagem do pé diabético a partir da ótica médica é escassa na literatura científica, o que, inclusive, dificultou a comparação com outros achados.

\section{AGRADECIMENTOS E FINANCIAMENTO}

Agradecemos ao Conselho Nacional de Desenvolvimento Científico e Tecnológico (CNPq) pela bolsa de Iniciação Científica, número de processo de bolsa 145161/2018-8, concedida para a realização da pesquisa.

\section{REFERÊNCIAS}

1. AALAA M, et al. Diabetic foot workshop: Improving technical and educational skills for nurses. Med J Islam Repub Iran 2017; 31(1): 1-3.

2. AL-RUBEAAN K, et al. All-cause mortality among diabetic foot patients and related risk factors in Saudi Arabia. PLoS One. 2017; 12(11): e0188097

3. AMORIM MMA, et al. Competences for self-care and self-control in diabetes mellitus type 2 in primary health care. World J Diabetes 2019; 10(8): 454-462.

4. BISPO JÚNIOR JP, MOREIRA DC. Núcleo de Apoio à Saúde da Família: concepções, implicações e desafios para o apoio matricial. Trab. Educ. Saúde 2018.16(2): 683-702.

5. BRASIL. Manual do pé diabético: estratégias para o cuidado da pessoa com doença crônica. 2016. Disponível em: http://189.28.128.100/dab/docs/portaldab/publicacoes/manual_do_pe_diabetico.pdf. Acessado em: 20 de junho de 2020.

6. CAMPOS CJG. Método de análise de conteúdo: ferramenta para a análise de dados qualitativos no campo da saúde. Rev. bras. enferm. 2004; 57(5): 611-614.

7. CRUZ PJSC, et al. Educação popular como orientadora de grupos de promoção à saúde de pessoas com hipertensão e diabetes na atenção básica: caminhos e aprendizados com base em uma experiência. Revista de Aps. 2018; 21(3): 387-398.

8. DUTRA LMA, et al. Avaliação do risco de ulceração em indivíduos diabéticos. Rev Bras Enferm. 2018;71(2):785-791.

9. FEITOSA MNL, et al. Assistência de enfermagem na atenção primária ao paciente com risco potencial de desenvolver pé diabético: uma revisão bibliográfica. Revista Uningá 2017; 54(1): 185-96.

10. FERNÁNDEZ IC, RUMBO-PRIETO JM. Riesgo de pie diabético y déficit de autocuidados en pacientes con Diabetes Mellitus Tipo 2. Enfermería Universitaria 2018, 15(1): 17-29.

11. FONTANELLA BJB, et al. Amostragem em pesquisas qualitativas: proposta de procedimentos para constatar saturação teórica. Cad Saude Publica 2011; 27(2): 388-94.

12. FONTANELLA BJB, et al. Amostragem por saturação em pesquisas qualitativas em saúde: contribuições teóricas. Cad Saude Publica 2008; 24(1): 17-27.

13. GAMA CAP, et al. Diabetes Mellitus e atenção primária: percepção dos profissionais sobre os problemas relacionados ao cuidado oferecido às pessoas com diabetes. Pesqui prat psicossociais. 2017; 12(3): e1398. 
14. GARBEY DLC, et al. Pie de riesgo en personas con diabetes mellitus de tipo 2 en la Atención Primaria de Salud durante 2016. MEDISAN 2018; 21(5): 518-530.

15. GOMES AC, et al. Adherence to pharmacological and nonpharmacological treatments in adults with type 2 diabetes. Mundo da Saúde 2020; 44: 381-396.

16. IDF. Diabetes Atlas. 2019. Disponível em: https://www.diabetes.org.br/publico/images/Atlas_IDF_2019.pdf. Acessado em: 18 de maio de 2020.

17. KUMARASINGHE SA, et al. Nurses' knowledge on diabetic foot ulcer disease and their attitudes towards patients affected: A cross-sectional institution-based study. J Clin Nurs. 2018; 27(1-2): 203-212.

18. LEITE RF. A perspectiva da análise de conteúdo na pesquisa qualitativa: algumas considerações. Revista Pesquisa Qualitativa 2017;5(9):539-51.

19. LUCOVEIS MLS, et al. Grau de risco para úlceras nos pés por diabetes: avaliação de enfermagem. Rev Bras Enferm. 2018; 71(6): 3217-3223.

20. MONTIEL-JARQUÍN AJ, et al. Costes directos de atención médica del pie diabético en el segundo nivel de atención médica. Revista Chilena de Cirurgía. 2017; 69(2): 118-23.

21. PEREIRA LF, et al. Ações do enfermeiro na prevenção do pé diabético: o olhar da pessoa com diabetes mellitus. J. res.: fundam. care. Online. 2017; 9(4): 1008-1014.

22. REYES-GARCíA R, et al. Documento de abordaje integral de la diabetes tipo 2. Endocrinol Diabetes Nutr. 2019; 66(7): 443-458.

23. SÁ SCM, et al. Desafios e potencialidade da atuação da equipe multiprofissional na atenção primária em saúde. Saúde Coletiva (Barueri). 2021; 11(61): 4918-4929.

24. SALCI MA, et al. Primary care for diabetes mellitus patients from the perspective of the care model for chronic conditions. Rev. Latino-Am. Enfermagem. 2017; 25: 2882.

25. SANTOS AL, et al. Adesão ao tratamento de diabetes Mellitus e relação com a assistência na Atenção Primária. REME - Rev Min Enferm. 2020; 24: e1279

26. SILVA AH, FOSSÁ MI. Análise de conteúdo: Exemplo de aplicação da técnica para análise de dados qualitativos. Qualitas Ver Eletrônica. 2015; 16(1).

27. SILVEIRA DM, et al. Pé Diabético: onde podemos intervir? HU Revista 2017; 43(1): 13-18.

28. SOMAYAJI R, et al. The impact of team based interprofessional comprehensive assessments on the diagnosis and management of diabetic foot ulcers: a retrospective cohort study. PLoS One. 2017; 12(9): e0185251

29. VAN-NETTEN JJ, et al. Prevention of foot ulcers in the at-risk patient with diabetes: a systematic review. Diabetes Metab Res Rev, 2016; 32(Suppl.1): 84-98. 